\title{
Phylogenetic Relationships of Three Porcine Mycoplasmas, Mycoplasma hyopneumoniae, Mycoplasma flocculare, and Mycoplasma hyorhinis, and Complete 16S rRNA Sequence of $M$. flocculare
}

\author{
G. W. STEMKE, ${ }^{1 *}$ F. LAIGRET, ${ }^{2}$ O. GRAU,$^{2}$ AND J. M. BOVÉ ${ }^{2}$
}

Department of Microbiology, University of Alberta, Edmonton, Alberta, T6G 2E9, Canada, ${ }^{1}$ and Laboratoire de Biologie Cellulaire et Moleculaire, Institut National de la Recherche Agronomique et Université de Bordeaux II, Domaine de la Grande Ferrade, 33883 Villenave d'Ornon Cedex, France ${ }^{2}$

\begin{abstract}
The nucleotide sequence of the 16S rRNA gene of Mycoplasma flocculare was determined and was compared with the sequence of a related porcine mycoplasma, Mycoplasma hyopneumoniae. While the overall level of DNA-DNA homology was approximately $11 \%$, sequence alignment of the two 16S rRNA genes yielded a homology value of more than $95 \%$, emphasizing the highly conserved nature of the 16S rRNA gene. Multiple sequence alignments with other mollicutes indicated that $M$. flocculare, M. hyopneumoniae, and Mycoplasma hyorhinis form a subcluster within the fermentans phylogroup, and this subcluster is distinct from the Mycoplasma pneumoniae phylogroup. Thus, the three mycoplasmas isolated from porcine respiratory systems exhibit phylogenetic similarities.
\end{abstract}

The following three mycoplasmal species have been isolated from porcine respiratory systems: Mycoplasma hyopneumoniae (Mycoplasma suipneumoniae), Mycoplasma flocculare, and Mycoplasma hyorhinis. Early work on the taxonomy of $M$. hyopneumoniae and $M$. flocculare was reported by Rose et al. (14). $M$. hyopneumoniae is a recognized etiological agent of porcine mycoplasmal pneumonia, while $M$. hyorhinis is an agent of polyserositis and arthritis (15). $M$. flocculare has not yet been implicated as an agent of naturally occurring diseases. However, antigenic cross-reactivity of $M$. flocculare and $M$. hyopneumoniae has been recognized; there is a weaker and more variable reaction with $M$. hyorhinis (1). The results of DNA-DNA hybridization studies have also indicated that there is some relatedness between $M$. hyopneumoniae and $M$. flocculare (10 to $11 \%$ ); in contrast, $M$. hyopneumoniae and $M$. flocculare cross-hybridize with nonporcine mycoplasmas at levels of about $3 \%$ (17).

Woese (21) and Weisburg et al. (20) have used analysis of 16S rRNA gene sequences to determine the phylogenetic and taxonomic relationships among Mollicutes genera and species, as well as between Mollicutes and bacteria. We determined the complete $16 \mathrm{~S}$ rRNA nucleotide sequence of $M$. flocculare and compared it with the previously published complete sequence of $M$. hyopneumoniae (19), as well as with partial sequences of several other Mollicutes, including $M$. hyorhinis, obtained from data banks.

\section{MATERIALS AND METHODS}

Culture. $M$. flocculare Ms42 was obtained from M. Kobish and was propagated on Friis medium (2) to the first visible color change: Cells were harvested by centrifugation at $14,000 \times g$ for $25 \mathrm{~min}$, washed once, and either used immediately for DNA preparation or stored as a frozen pellet at $-80^{\circ} \mathrm{C}$ until they were used. DNA was purified as described previously (5). $M$. flocculare Ms42 (= ATCC 27716) was grown similarly, and its DNA was prepared.

\footnotetext{
* Corresponding author.
}

The authenticity of the strain obtained from M. Kobish was determined by comparing the DNA of this strain with DNA obtained from $M$. flocculare Ms42 (= ATCC 27716); identical restriction enzyme digests were observed with both $E c o$ RI and HindIII. These patterns differed from those of $M$. hyopneumoniae and $M$. hyorhinis. Furthermore, identical banding patterns for both samples of $M$. flocculare DNA were detected by using a $5 \mathrm{~S}$ rRNA oligonucleotide probe. In addition, a 16S rRNA gene probe reacted with two EcoRI fragments that were approximately 5,700 and 6,500 bp long with both DNA samples, differing also from the sizes of $16 \mathrm{~S}$ rRNA banding fragments found with either $M$. hyopneumoniae or M. hyorhinis.

Data base sequences. Sequences for several Mollicutes $16 \mathrm{~S}$ rRNAs were obtained from the GenBank data base.

Vectors and hosts. Lambda-Dash EcoRI (Stratagene, La Jolla, Calif.) (EcoRI-cut and alkaline phosphatase-treated arms) was used with the host Escherichia coli LE392. We also used plasmid Bluescript (Stratagene) with strain LE392 as the host.

Cloning. In the cloning experiments first the phage arms were mixed with large fragments $(9$ to $23 \mathrm{~kb}$ ) of a partial EcoRI digest of $M$. flocculare genomic DNA which had been size fractionated on sucrose as described elsewhere (16). The arms and insertion were ligated and packaged into phage heads by using a commercial packaging extract (Gigapack; Stratagene) and the supplier's protocol. This material was then plated with excess host bacteria and incubated until plaques were visible. Nitrocellulose membranes were applied to allow phage to adsorb to the membranes; these membranes were then were treated with alkali, neutralized, blocked, and probed for Mollicutes 16S rRNA sequences by using an isolated radiolabeled insertion from Spiroplasma citri (9). The membranes were exposed to X-ray film and aligned with the phage plaque plate to pick positive plaques; six of these were plaque purified three times, each time verifying the $16 \mathrm{~S}$ rRNA insertion by using the $16 \mathrm{~S}$ rRNA probe.

While we encountered difficulties in the preparation of high-titer phage lysates, multiple rounds of plate lysates 
resulted in the preparation of adequate phage to purify and utilize for DNA preparation.

Attempts to directly sequence the lambda DNA insertion by using the double-strand sequencing procedure (see below) gave variable results. The insertion was then subcloned into Bluescript; a partial EcoRI digest was directly cloned into the EcoRI site of this plasmid. Colonies appearing on Luria broth plates containing $50 \mu \mathrm{g}$ of ampicillin per ml were picked, and plasmid minipreparations were obtained. These preparations were again probed with the $S$. citri $16 \mathrm{~S}$ rRNA insertion, and six were chosen for further work. One, pF1-14, was initially chosen for sequencing (see below). When we subsequently found that the entire 16S rRNA gene was not present in the pF1-14 insertion, we used pF1-6, which had a larger insertion. pF1-14 had a 6.5 -kb insertion, and $\mathrm{pF} 1-6$ had a $12-\mathrm{kb}$ insertion. The sequence which we determined was based upon data from both plasmids.

Sequencing. We used commercial dideoxy sequencing kit (Pharmacia, Uppsala, Sweden) and the Pharmacia protocol for double-strand sequencing. The initial primers were three universal 16S rRNA oligonucleotides (11). The resulting sequence was used to prepare additional primers for primer extension and fill-in, as well as to sequence from the other DNA strand.

Sequencing gels containing $6 \%$ acrylamide in $6 \mathrm{M}$ urea were prepared and run as described previously (16). The gels were fixed in methanol-acetic acid for autoradiography with ${ }^{35} \mathrm{~S}$ and were read by using an IBI sonic digitizer (International Biotechnologies, Inc., New Haven, Conn.) or a DNAParrot cursor and software ( $T \&$ t Research, Etobicoke, Ontario, Canada).

Analysis. Sequencing files were compared by using the NIH/DCRT analysis system of M. Kanehisa (IBM PC version).

Multiple sequence comparisons were done by using the PCGENE (IntelliGenetics, Inc., Mountain View, Calif.) subroutine Clustal, which also provides a dendrogram output. Sequences need to be truncated to 1,200 nucleotides for this program. The default truncation is from the rRNA 5' end. Qualitatively identical results were obtained when we used files truncated from the 3 ' end, and the results were not changed significantly by decreasing the gap penalty from default values. The IntelliGenetics Suite program (IntelliGenetics, Inc.) adapted for the UNIX system on a Sun Workstation was used for full-length sequence comparisons.

Nucleotide sequence accession numbers. The nucleotide sequence described below has been deposited in the EMBL data base and is available under accession number X63377. The GenBank nucleotide sequence accession numbers for other Mollicutes 16S rRNAs which we used are as follows: M. hyopneumoniae, M30378; M. hyorhinis, M24658; Mycoplasma arginini, M24579; Mycoplasma fermentans, M24289; Mycoplasma hominis, M24473; Mycoplasma capricolum, X00921; Mycoplasma pneumoniae, M29061; Mycoplasma muris, M23939; Mycoplasma salivarium, M24661; Spiroplasma apis, M23937; and Clostridium innocuum, M23732.

\section{RESULTS AND DISCUSSION}

The complete nucleotide sequence of the $M$. flocculare 16S rRNA gene is shown in Fig. 1 along with the sequences of two porcine mycoplasmas, $M$. hyopneumoniae and $M$. hyorhinis, and Mycoplasma arginini and Mycoplasma muris, two less closely related mycoplasmas. The presumptive starting and terminating nucleotides for the mature $16 \mathrm{~S}$
rRNA were chosen on the basis of the sequence of $M$. hyopneumoniae $16 \mathrm{~S}$ rRNA. This gave a full-length sequence of 1,548 nucleotides, making it among the largest known mycoplasma sequences. The very highly conserved internal EcoRI site (4) at position +690 (counting from the mature 16S rRNA 5' site) was noted.

While the overall level of DNA-DNA homology between $M$. hyopneumoniae and $M$. flocculare is only about $11 \%$ (17), the sequence analysis program of Kanehisa indicated that there is $95.6 \%$ homology with the sequence reported for $M$. hyopneumoniae (19) within the rRNA coding region. Thus, the highly conserved nature of the 16S rRNA gene is further documented. Most of the variations and the variations that were most closely spaced occurred in the region between positions 64 and 103, which represents a non-hydrogenbinding region in the stem-loop structure of $M$. capricolum $16 \mathrm{~S}$ rRNA (7). Multiple variations also occurred in the regions from position 193 to position 231 and from position 478 to position 500 .

The Clustal multiple-alignment program, in which we used the complete 16S rRNA gene of $M$. hyopneumoniae and complete and partial sequences downloaded from the GenBank data base, gave the dendrogram shown in Fig. 2 again indicating the close relationship between $M$. flocculare and $M$. hyopneumoniae but also indicating that these two species represent a subcluster of a larger cluster that includes $M$. hyorhinis. This larger cluster in turn is a portion of the cluster that includes $M$. fermentans, $M$. hominis, and $M$. arginini. This cluster is deeply separated from the cluster containing M. muris, M. pneumoniae, and Ureaplasma urealyticum. The clustering of ureaplasma with $M$. pneumoniae and the clustering of $M$. capricolum with a spiroplasma are in agreement with the results of Laigret et al. (10). Clostridium innocuum was used as an outgroup. Thus, our findings both confirm the findings of Woese (21), Weisburg et al. (20), and Laigret et al. (10) and provide support for the relationships described above for the porcine mycoplasmas.

Figure 1 shows the multiply aligned sequences and demonstrates the variations that are indicative of some of the cluster separations. Particularly notable are the $M$. flocculare-M. hyopneumoniae sequence $\mathrm{AAA}(\mathrm{G} / \mathrm{A}) \mathrm{TA}$ at position 96 and the CT sequence at position 494. The M. fermentans cluster, to which these species belong, has a 2-base deletion at position 190 and a 3- or 4-nucleotide deletion compared with the $M$. muris-ureaplasma group at position 835 . $M$. flocculare has 3- and 5-base additions at position 79 compared with $M$. hyopneumoniae and $M$. hyorhinis, respectively.

Conclusion. We determined the complete 16S rRNA nucleotide sequence of $M$. flocculare and compared it with the previously published complete sequence of $M$. hyopneumoniae, as well as with partial sequences of several other mollicutes, including $M$. hyorhinis, obtained from data banks. The very close sequence homology of $M$. hyopneumoniae and $M$. flocculare gives very firm evidence of the close phylogenetic relationship of these organisms and explains the antigenic cross-reactions observed between these organisms.

In the $3^{\prime}$-end region of both $M$. hyopneumoniae and $M$. flocculare the identical sequence GAACACCTCCTTTCTA is found. This sequence has been shown experimentally to be the 3'-OH end of the 16S rRNA of $M$. hyopneumoniae (17), and because of the identity of the DNA sequence in $M$. flocculare it may be postulated that the $3^{\prime}$ end is the same. This sequence is identical to the sequence in Bacillus subtilis (12) and to the sequence in Mycoplasma iowae (3). Other 


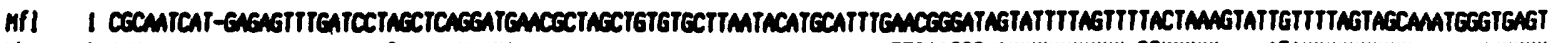

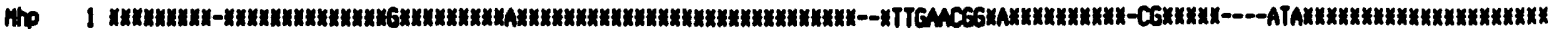

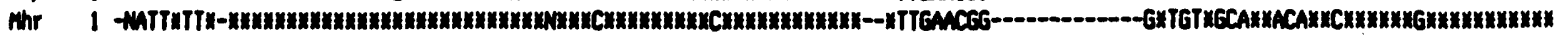

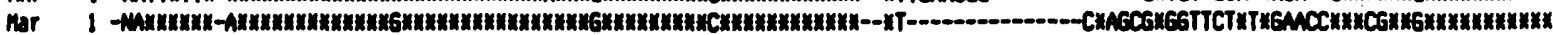

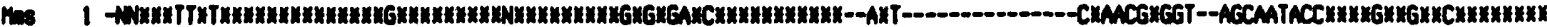

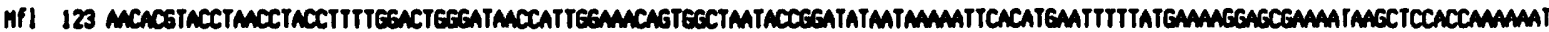

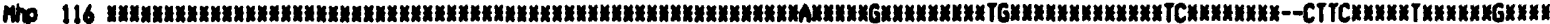

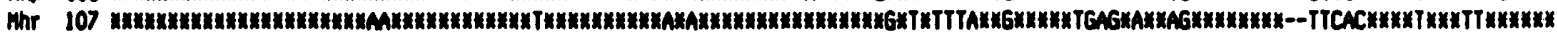

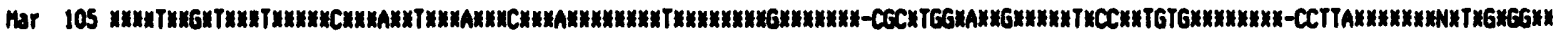

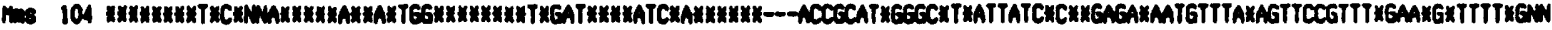

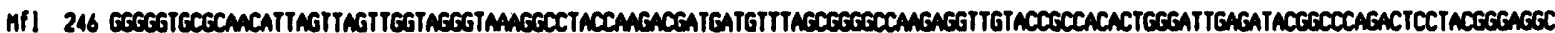

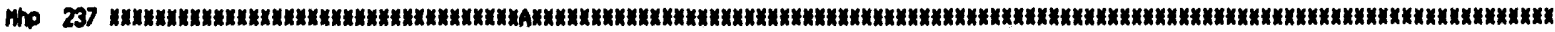

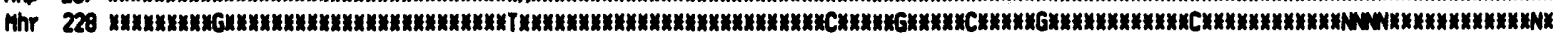

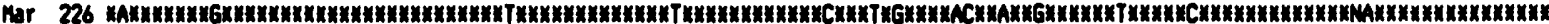

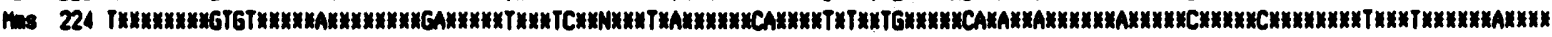

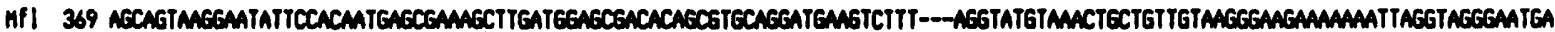

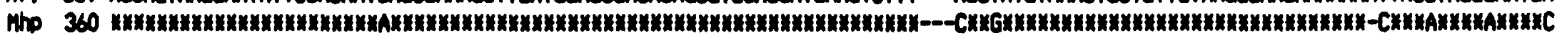

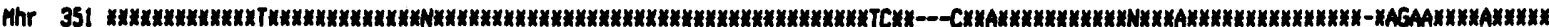

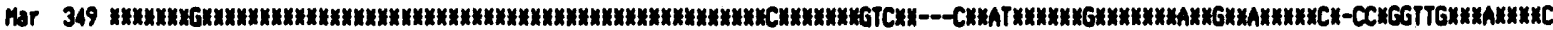

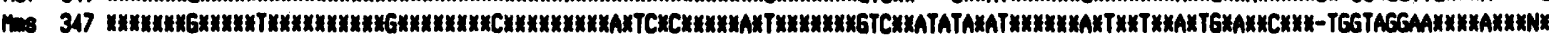

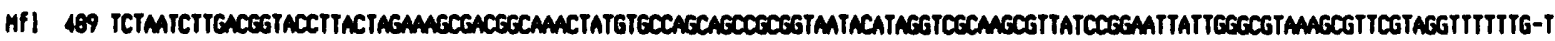

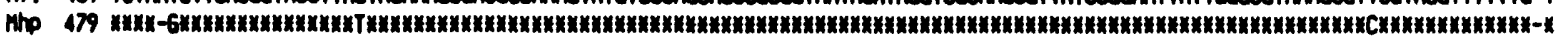

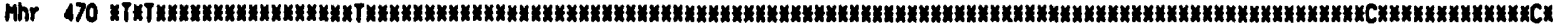

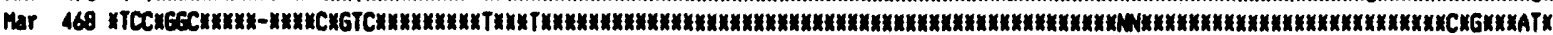

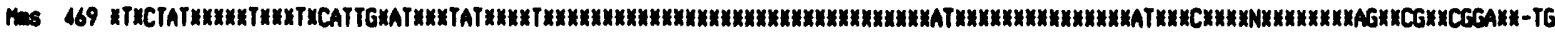

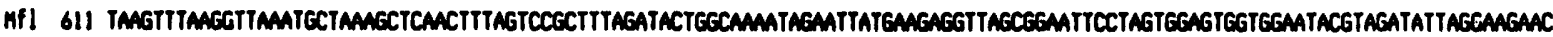

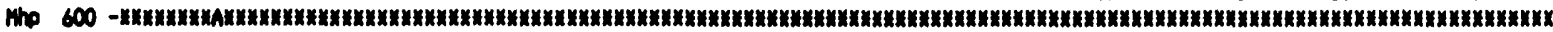

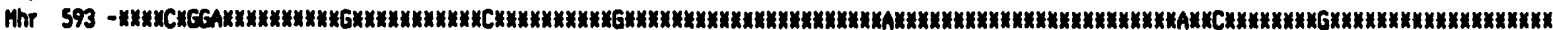

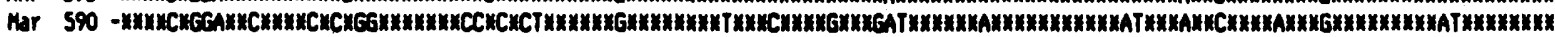

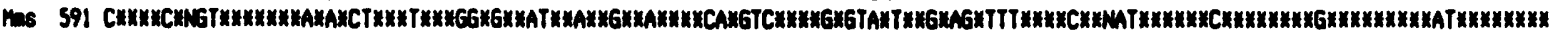

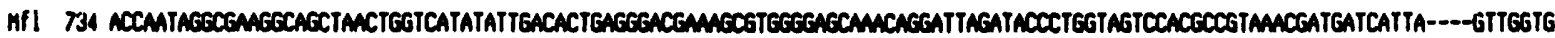
Tho 722 й

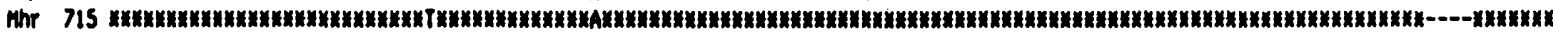

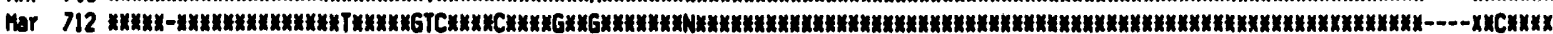
Thง 714 хихи-

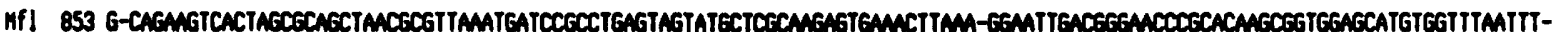

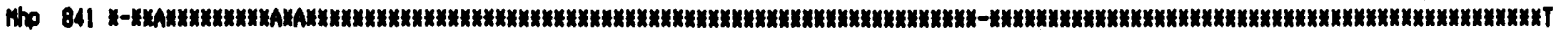

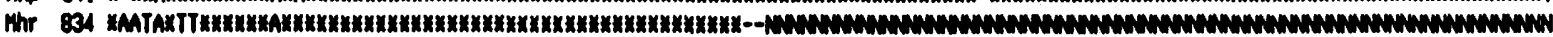

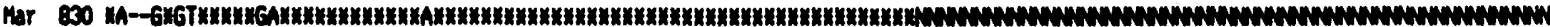

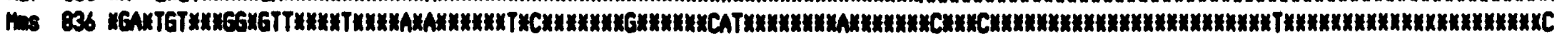

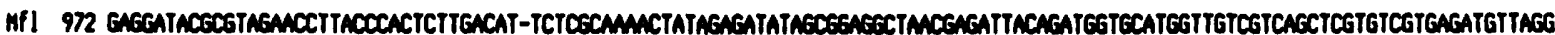

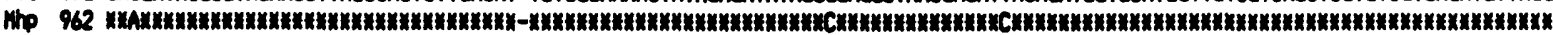

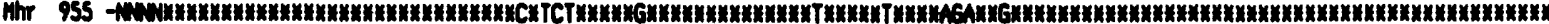

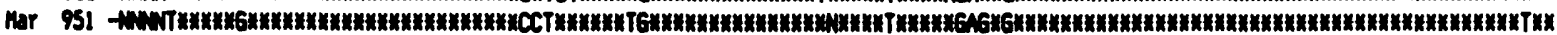

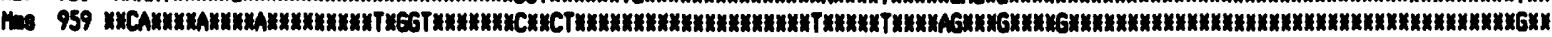

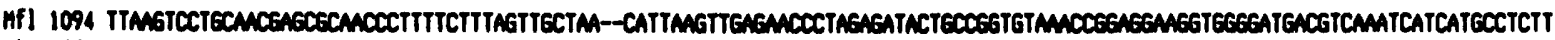

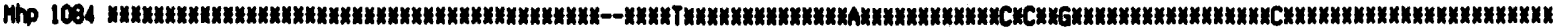

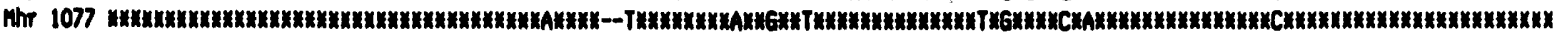

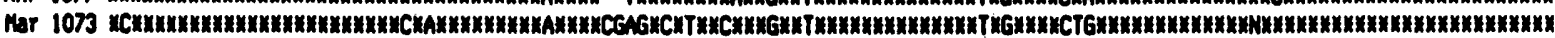

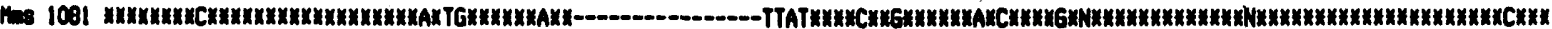

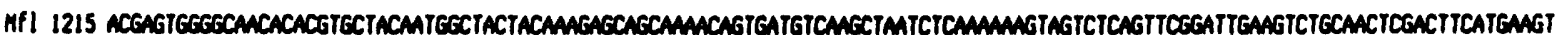

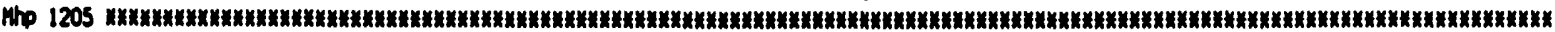

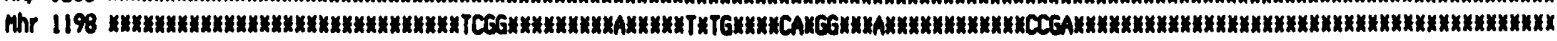

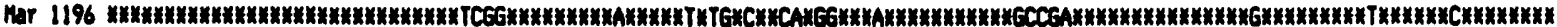

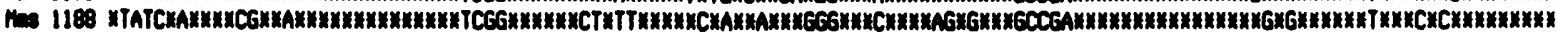

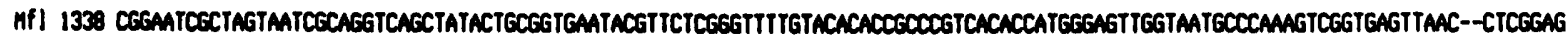
The 1328 ำ

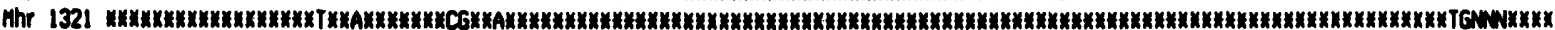

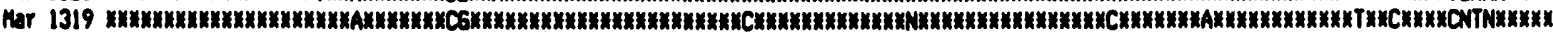

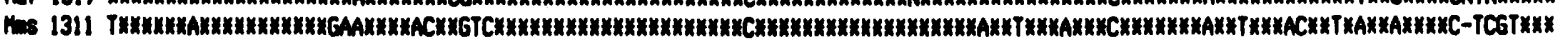

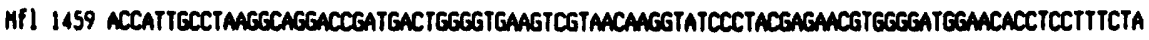

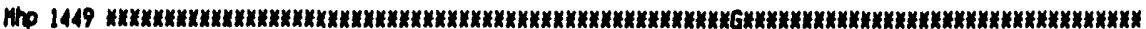

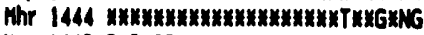

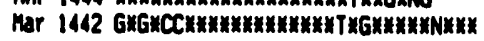

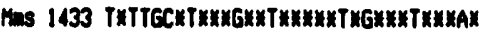

FIG. 1. Nucleotide sequence of $M$. flocculare and sequence alignment with other representative Mycoplasma species. An asterisk indicates a nucleotide that was identical to the $M$. flocculare nucleotide; a dash indicates a gap of one nucleotide. N, undetermined nucleotide; Mfl, M. flocculare; Mhp, M. hyopneumoniae; Mhr, M. hyorhinis; Mar, M. arginini; Mms, M. muris. 


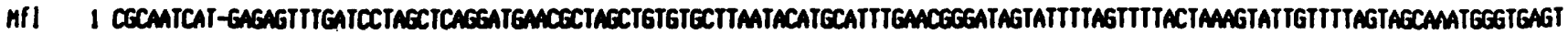

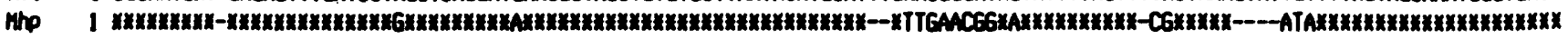

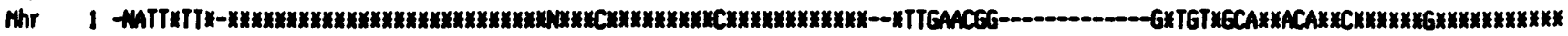

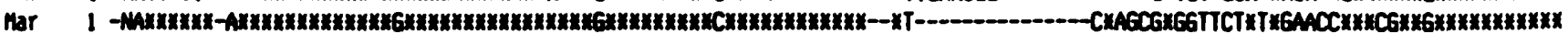
Me 1 -

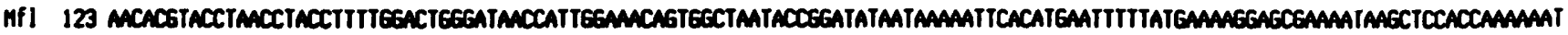

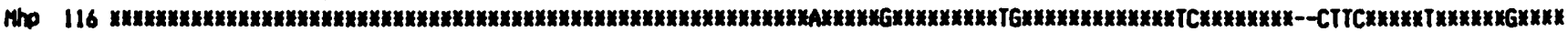

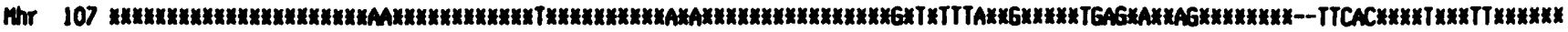

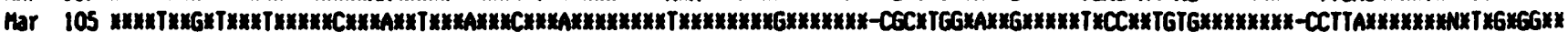

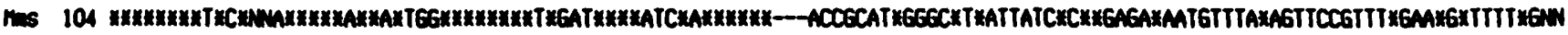

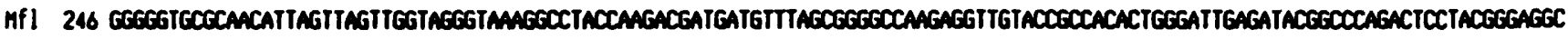

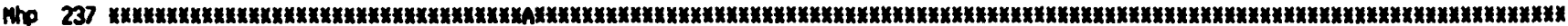

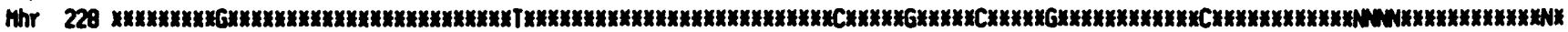

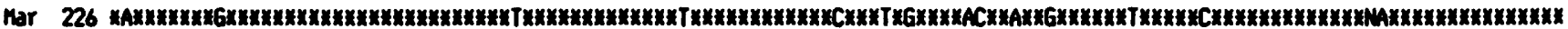

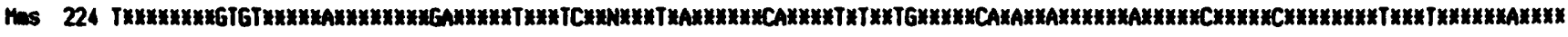

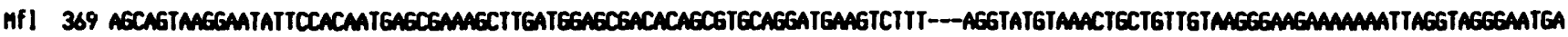

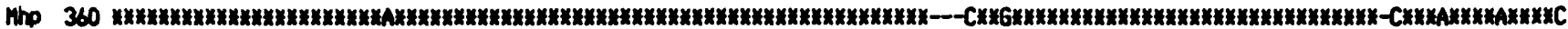

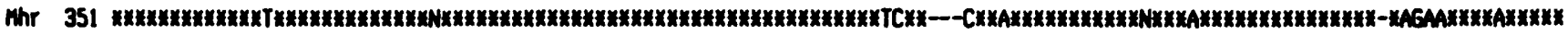

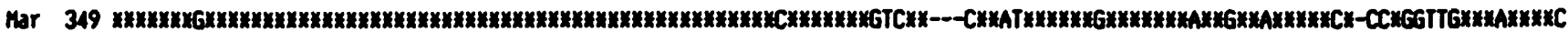

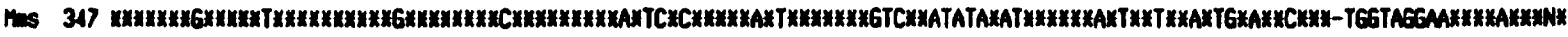

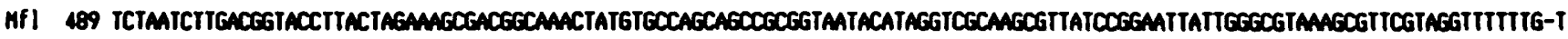

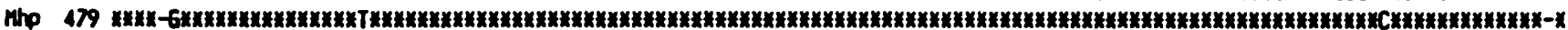

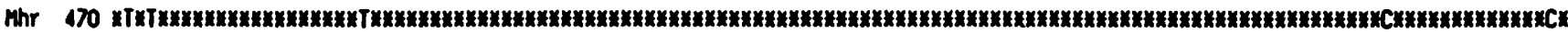

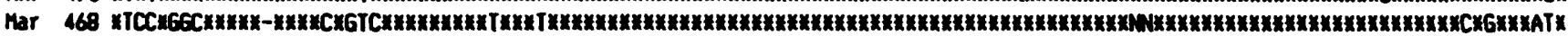

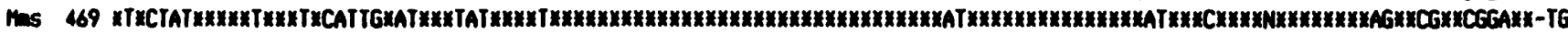

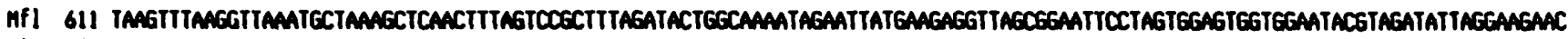

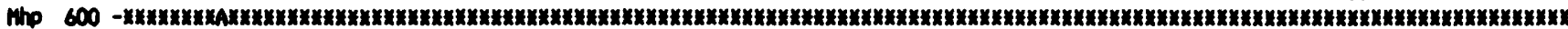

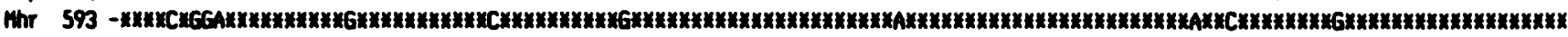

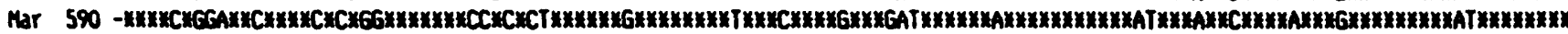

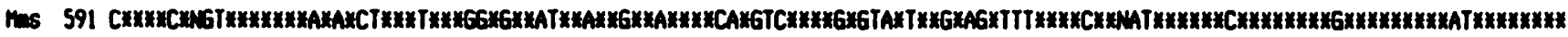

Mfl 734 a

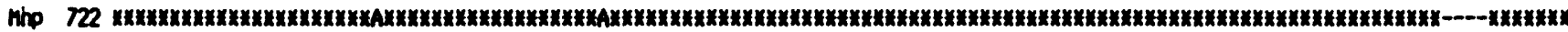

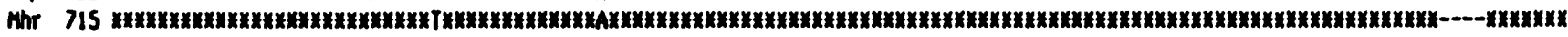

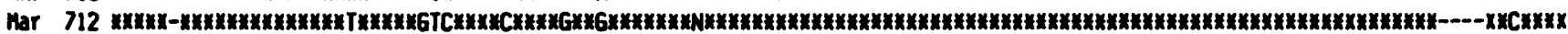

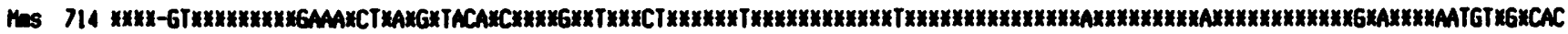

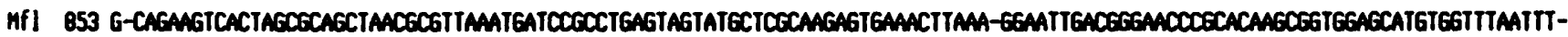

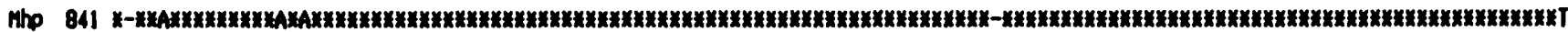

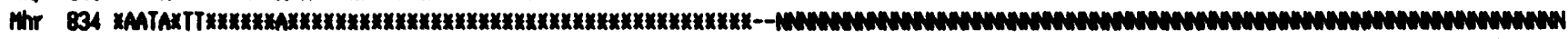

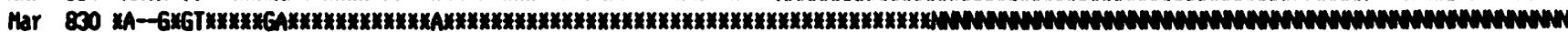

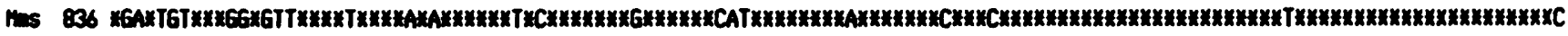

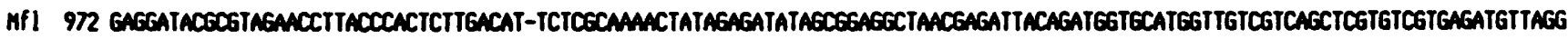

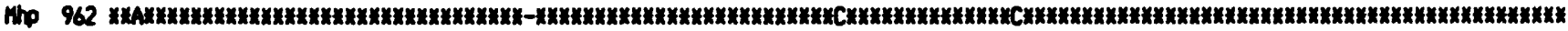
Th 955 -

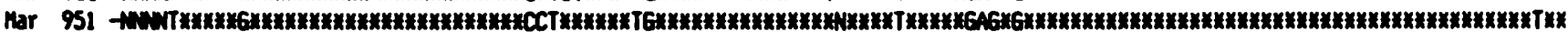

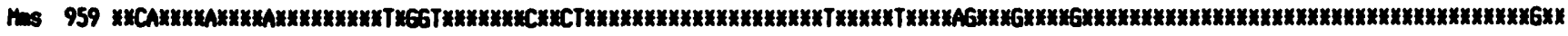

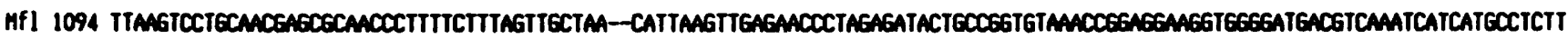

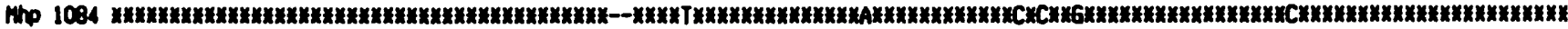

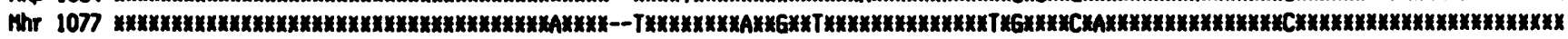

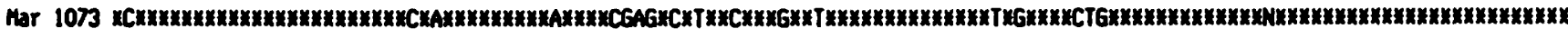

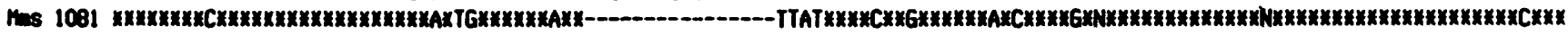

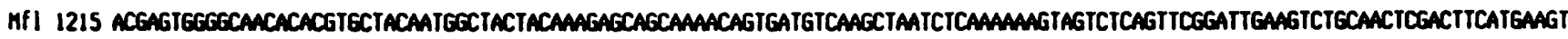

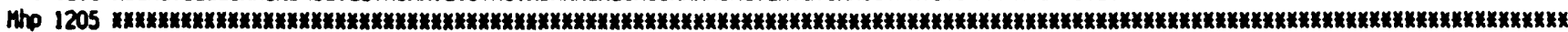

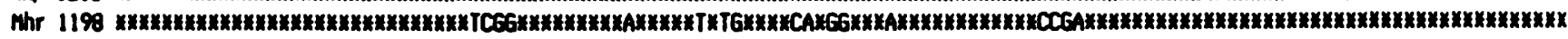

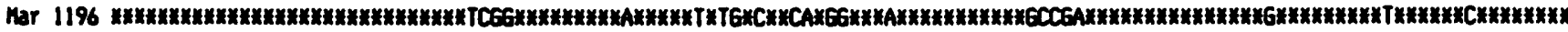

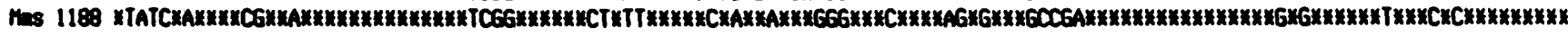

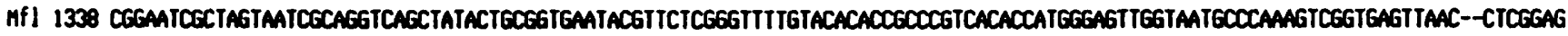

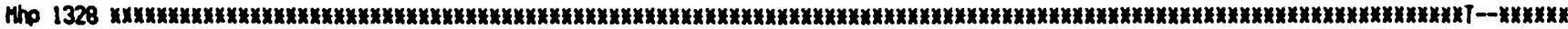
Thr 1321 ต

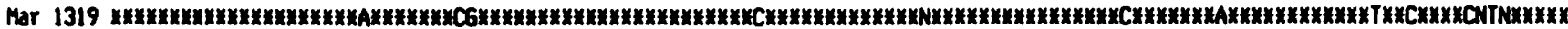

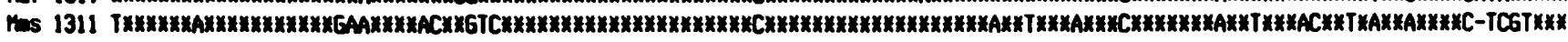

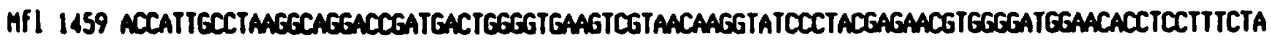

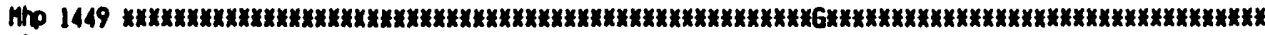

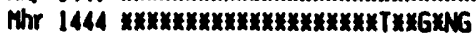

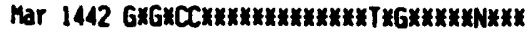

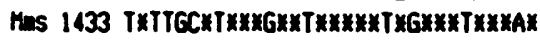




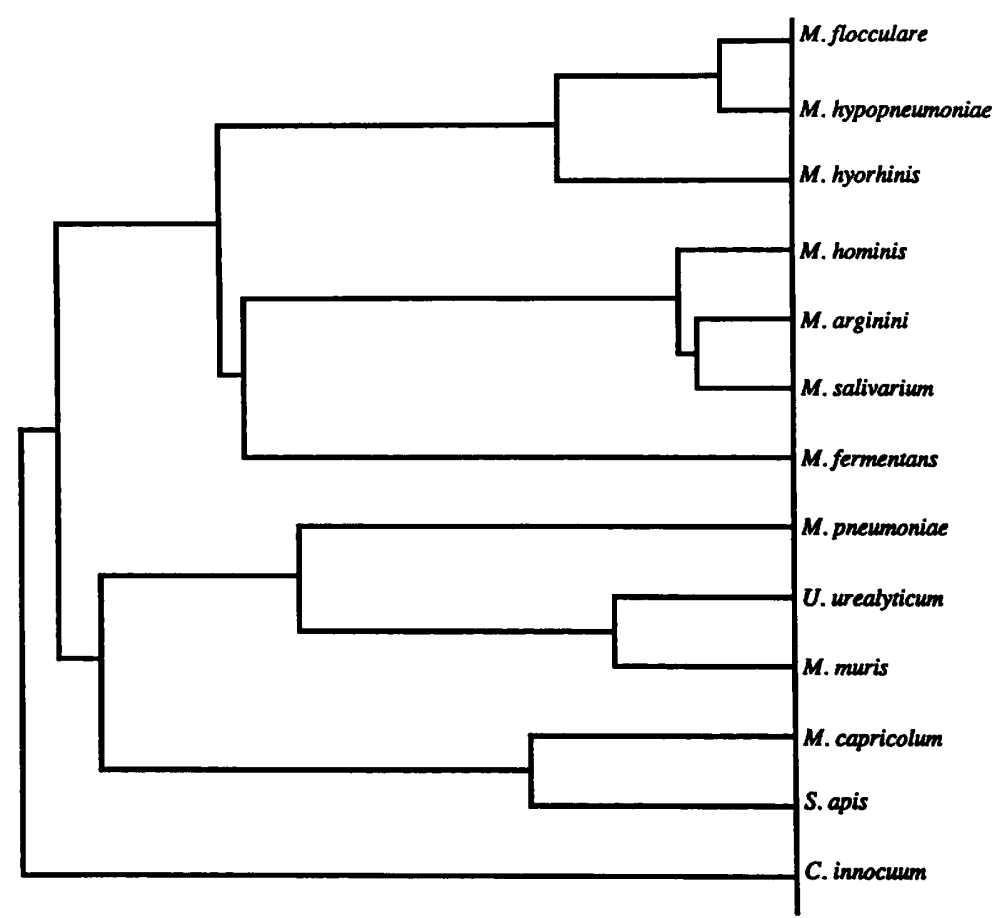

FIG. 2. Dendrogram representing the phylogenetic relationships derived from 16S rRNA sequence homologies of Mollicutes species. Sequences for comparison with $M$. flocculare were obtained from GenBank. The PCGene multiple-alignment subroutine Cluster was used to generate the dendrogram. All of the sequences were truncated to 1,200 nucleotides for this comparison.

mollicutes belonging to the spiroplasma phylogroup have a slightly longer $3^{\prime}$ end.

While the 16S rRNA of $M$. flocculare is larger than that of $M$. hyopneumoniae, it is not immediately evident that $M$. flocculare is ancestral to $M$. hyopneumoniae or vice versa. Thus, the pattern of changes in $M$. hyopneumoniae, $M$. flocculare, and $M$. hyorhinis does not clearly indicate a direction of change. For instance, $M$. hyopneumoniae and $M$. hyorhinis have adenine at position 488, while $M$. flocculare has cytosine; however, $M$. flocculare and $M$. hyorhinis have adenine at position 240 , while $M$. hyopneumoniae has guanine.

$M$. hyopneumoniae has a single set of rRNA genes, and the $5 \mathrm{~S}$ gene is separated by more than $4 \mathrm{~kb}$ from the $16 \mathrm{~S}-23 \mathrm{~S}$ rRNA genes (19). We found (6) that $M$. flocculare also has only a single set of rRNA genes, on the basis of the results of restriction endonuclease digestion with a variety of enzymes; in no case was 5S and 16S rRNA activity detected on a single fragment with probes specific for these genes. We are in the process of constructing a physical map of the $M$. flocculare genome in which we will show that the 5S rRNA gene of both $M$. hyopneumoniae and $M$. flocculare is at least $100 \mathrm{~kb}$ from the $16 \mathrm{~S}$ gene.

We also found (6) by performing a restriction ribonuclease analysis that $M$. hyorhinis probably has only one set of rRNA genes; also, $5 \mathrm{~S}$ and $16 \mathrm{~S}$ gene probes never reacted with the same restriction fragment. These findings further indicate the close genetic relationship between the three mycoplasmas isolated from porcine respiratory systems. In turn, this suggests that these organisms evolved from a common ancestor in a common host. We have reported previously (17) that $M$. hyopneumoniae and $M$. flocculare have identical genomic $\mathrm{G}+\mathrm{C}$ contents (33 mol\%); more recently, Kirchhoff and Flossdorf reported that $M$. hyopneu- moniae has a $\mathrm{G}+\mathrm{C}$ content of $27.5 \mathrm{~mol} \%$ (8). $M$. hyorhinis has been reported to have a $\mathrm{G}+\mathrm{C}$ content of $28 \mathrm{~mol} \%$ (13). Redetermination of these values will be required to ascertain whether any of them might be in error or whether the possible variation between $M$. hyopneumoniae-M. flocculare and $M$. hyorhinis simply reflects the plasticity of Mollicutes genomes.

\section{ACKNOWLEDGMENTS}

We thank M. Kobisch for providing the strain Ms42 culture and J. A. Robertson for growing and harvesting the mycoplasma.

A portion of this work was done while G.W.S. was on study leave at the Institute National de la Recherche Agronomique and was partially supported by a fellowship from this institute. Additional research support was provided by grant OG0007320 from the National Sciences and Engineering Research Council of Canada.

\section{REFERENCES}

1. Freeman, M. J., C. H. Armstrong, L. L. Sands-Freeman, and M. Lopez-Osuna. 1984. Serological cross-reactivity of porcine reference antisera to Mycoplasma hyopneumoniae, M. flocculare, $M$. hyorhinis and $M$. hyosynoviae indicated by the enzymelinked immunosorbent assay, complement fixation and indirect hemagglutination tests. Can. J. Comp. Med. 48:202-207.

2. Friis, N. F. 1975 . Some recommendations concerning primary isolation of Mycoplasma suipneumoniae and Mycoplasma flocculare. Nord. Veterinaermed. 27:337-339.

3. Grau, O. 1991. Analyse des gènes ribosomique des mollicutes, application à l'identitification d'un mollicute non classé et conséquences taxonomiques. Ph.D. thesis. Université de Bordeaux II, Bordeaux, France.

4. Grau, O., F. Laigret, and J. M. Bové. 1990. Analysis of ribosomal RNA genes in two spiroplasmas, one acholeplasma and one unclassified mollicute, p. 895-897. In G. Stanek, G. H. Cassell, J. G. Tully, and R. F. Whitcomb (ed.), Recent advances in mycoplasmology. Gustav Fischer Verlag, Stuttgart. 
5. Gross-Bellard, M., P. Oudet, and P. Chambon. 1973. Isolation of high molecular weight DNA from mammalian cells. Eur. J. Biochem. 36:32-38.

6. Huang, Y., and G. W. Stemke. 1989. Mycoplasma flocculare has a rRNA gene organization similar to Mycoplasma hyopneumoniae, abstr. G-32, p. 153. Abstr. 89th Annu. Meet. Am. Soc. Microbiol. 1989, American Society for Microbiology, Washington, D.C.

7. Iwami, M., A. Muto, F. Yamao, and S. Osawa. 1884. Nucleotide sequence of the $r m B$ 16S ribosomal RNA gene from $M y c o$ plasma capricolum. Mol. Gen. Genet. 196:317-322.

8. Kirchhoff, H., and J. Flossdorf. 1987. Determination of the guanine-plus-cytosine content of Mollicutes by isopycnic gradient centrifugation. Int. J. Syst. Bacteriol. 37:454-455.

9. Laigret, F., O. Grau, and J. M. Bové. 1990. Comparison of $16 \mathrm{~S}$ rDNA sequences of various mollicutes, p. 435-440. In G. Stanek, G. H. Cassell, J. G. Tully, and R. F. Whitcomb (ed.), Recent advances in mycoplasmology. Gustav Fischer Verlag, Stuttgart.

10. Laigret, F., K. J. Hackett, R. F. Whitcomb, J. G. Tully, and J. M. Bové. 1990. Ecology and phylogenetics of spiroplasmas: a short review, p. 55-59. In R. Lesel (ed.), Microbiology in poekilotherms. Elsevier Science Publications, Amsterdam.

11. Lane, D. J., D. Pave, G. J. Olsen, D. A. Stahl, M. L. Sogin, and N. R. Pace. 1985. Rapid determination of 16 S ribosomal RNA sequences for phylogenetic analysis. Proc. Natl. Acad. Sci. USA 82:6955-6985.

12. Loughney, K., E. Lund, and J. E. Dahlberg. 1983. Ribosomal RNA precursors of Bacillus subtilis. Nucleic Acids Res. 11: 6709-6721.

13. Razin, S., and E. A. Freundt. 1984. The mycoplasmas, p. 740-793. In N. R. Krieg and J. G. Holt (ed.), Bergey's manual of systematic bacteriology, vol. 1. The Williams \& Wilkins Co., Baltimore.
14. Rose, D. L., J. G. Tully, and R. G. Wittler. 1979. Taxonomy of some swine mycoplasmas: Mycoplasma suipneumoniae Goodwin et al. 1965, a later, objective synonym of Mycoplasma hyopneumoniae Mare and Switzer 1965; and the status of Mycoplasma flocculare Meyling and Friis 1972. Int. J. Syst. Bacteriol. 29:83-91.

15. Ross, R. F. 1982. Mycoplasmal diseases, p. 535-549. In A. D. Leman, R. D. Glock, W. L. Mengeling, R. H. C. Penny, E. Scholl, and B. Straw (ed.), Diseases of swine, 5th ed. Iowa State University Press, Ames.

16. Sambrook, J., E. F. Fritsch, and T. Maniatis (ed.). 1989. Molecular cloning, a laboratory manual, 2nd ed. Cold Spring Harbor Laboratory Press, Cold Spring Harbor, N.Y.

17. Stemke, G. W., D. J. McIntyre, K. L. Roy, M. E. Stemler, and J. A. Robertson. 1985. Guanine-plus-cytosine compositions of and deoxyribonucleic acid hybridization comparisons between Mycoplasma hyopneumoniae and Mycoplasma flocculare. Int. J. Syst. Bacteriol. 35:527-529.

18. Taschke, C., and R. Herrmann. 1986. Analysis of transcription and processing signals of the 16S-23S rRNA operon of Mycoplasma hyopneumoniae. Mol. Gen. Genet. 205:434-441.

19. Taschke, C., J. Kinkert, and R. Herrmann. 1986. Organization of the ribosomal RNA genes in Mycoplasma hyopneumoniae: the 5S rRNA gene is separated from the $16 \mathrm{~S}$ and $23 \mathrm{~S}$ genes. Mol. Gen. Genet. 205:428-433.

20. Weisburg, W. G., J. G. Tully, D. L. Rose, J. P. Petzel, H. Oyaizu, D. Yang, L. Mandelco, J. Sechrest, T. G. Lawrence, J. Van Etten, J. Maniloff, and C. R. Woese. 1989. A phylogenetic analysis of the mycoplasmas: basis for their classification. J. Bacteriol. 171:6455-6467.

21. Woese, C. R. 1987. Bacterial evolution. Microbiol. Rev. 51:221271. 